\title{
Comparison of Intraoperative Outcomes between Single-incision Robotic Cholecystectomy and Multi-incision Robotic Cholecystectomy
}

\author{
Philip Schertz ${ }^{1}$, Subhasis Misra ${ }^{1}$, David Livert ${ }^{2}$, Joanne Mulligan ${ }^{2}$, Chand Rohatgi ${ }^{2}$ \\ 1. Surgery, Brandon Regional Hospital, Brandon, USA 2. Surgery, Easton Hospital, Easton, USA
}

Corresponding author: Philip Schertz,phil@drschertz.com

\section{Abstract}

\section{Background}

The aim of this study was to evaluate the differences in the key surgical factors for single-incision robotic cholecystectomy (SIRC) and multi-incision robotic cholecystectomy (MIRC).

\section{Methods}

A retrospective data review from August 2013 to April 2018 consisting of 104 SIRC and 105 MIRC cases was done considering factors including patient gender, age, operating time (skin incision to skin closure), robotic console time (docking to undocking), the preoperative diagnosis for surgery, any complications in surgery, length of stay (LOS), and estimated blood loss (EBL). Procedures with conversion away from original robotic cholecystectomy approach were excluded. Chi-square analysis ( $p$-value: 0.05 ) was run between the two data sets.

\section{Results}

A total of 209 robotic cholecystectomy cases were reviewed since 2013 . We found significantly less time with single-incision compared to multi-incision (single incision $=94.0$ minutes, multi-incision $=99.9$ minutes, $p=$ 0.016 ) and EBL (single-incision $=11.52 \mathrm{~mL}$, multi-incision $=17.17 \mathrm{~mL}, p=0.004$ ). There was no significant difference in age or robotic console time. The most common indication was symptomatic cholelithiasis overall, with equal cases of dyskinesia in single-incision approach, although there was no significant difference in indication between the two approaches. Intraoperatively, there was marginally significant use of irrigation in multi-incision (multi-incision 45 [42.9\%], single-incision 31 [29.8\%], $p=0.0499$ ) and no difference in Firefly, perforation, or intraoperative cholangiogram use. LOS results showed significant decreased stay in SIRC cases (single-incision 84 outpatients [80.8\%], multi-incision 75 [71.4\%]; $p=0.0379$ ).

\section{Conclusions}

Received 07/10/2019 Review began 08/06/2019 Review ended 08/10/2019 Published 08/13/2019

\section{๑) Copyright 2019}

Schertz et al. This is an open access article distributed under the terms of the Creative Commons Attribution License CC-BY 3.0., which permits unrestricted use, distribution, and reproduction in any medium, provided the original author and source are credited.
SIRC and MIRC are both safe and feasible ways to remove the inflamed/dysfunctional gallbladder. SIRC is associated with less operative time, less blood loss, and shorter hospital stay.

\section{Categories: Gastroenterology, General Surgery}

Keywords: robotic surgery, minimally invasive, robotic cholecystectomy

\section{Introduction}

The da Vinci system (Intuitive Surgical, Inc., Sunnyvale, CA) has been used to perform cholecystectomies since 1997, and single-incision robotic cholecystectomy (SIRC) was introduced in 2011 to overcome the previous limitations from a multi-incision robotic cholecystectomy (MIRC) [1-2]. SIRC has been favored in cholecystectomies because of the minimal scarring visible by hiding the entry point in the umbilicus and reduction in postoperative pain [3]. As an alternative to MIRC, SIRC has foreseeable benefits, but the real clinical benefits for patients remain a matter of debate. The primary pitfalls of SIRC have been associated with incisional/trocar site hernias and visceral injuries related to the larger midline incision $[2,4]$. SIRC has improved cosmetic appearance although the intraoperative difficulty is higher compared to MIRC, which may not offset the benefit especially in pediatrics [5]. Given the limited research, this study attempts to compare SIRC and MIRC cases in a retrospective approach at a single-community hospital across nine surgeons.

\section{Materials And Methods}

A retrospective review of operative records of 104 SIRC and 105 MIRC cases from August 2013 until April 2018 was performed. We collected data from all patients receiving robotic cholecystectomies during this time. The same standardized surgical technique was used for all patients and all operations were performed in house by nine different surgeons. Our data collection included the following variables: patient gender, 
age, operating time (skin incision to skin closure), robotic console time (docking to undocking), the preoperative diagnosis for surgery, any complications in surgery, length of stay (LOS), and estimated blood loss (EBL). We compared the outcomes of the two methods based on these variables. Exclusion criteria included any patients who underwent intra-operative conversion from robotic to either laparoscopic or open surgery; these included four single-incision and six multi-incision over the time frame of the study. We were unable to obtain data prior to 2014, and the most recent cases over the last six months did not have console time recorded. A literature review prior to data collection correlated our data collection with previous comparisons of robotic surgery techniques.

Statistical analysis measured means and standard deviations. Chi-square test was done. $P$-values of $<0.05$ were considered to represent statistically significant differences in data. The significance was used to prove or disprove the null hypothesis that there was no difference in outcomes and indications in MIRC and SIRC. This study was approved by the Easton Hospital's institutional Review Board.

\section{Surgical techniques}

Single-incision Robotic Cholecystectomy

The patient was brought to the operating room and positioned in the reverse Trendelenburg position with arms tucked at the sides. Nursing staff, a resident physician, and often a physician assistant trained in the robotic platform was present. A midline incision was made through the umbilicus with a single-site port placed with the target anatomy facing the camera. The robotic arms were placed over the patient with the robot resting by the patient's right shoulder. An assistant port was inserted and used to retract the gallbladder cephalad, while the triangle of Calot was dissected. After achieving the critical view of safety, the cystic duct and cystic artery were clipped and divided. The gallbladder was dissected off the gallbladder fossa and removed with the umbilical port once the dissection was finished. If there was bile spillage, the fossa was irrigated until clear irrigant was suctioned before removing the port. The fascia defect was closed.

Multi-incision Robotic Cholecystectomy

The approach is similar to the single-incision approach with the exception that a single midline incision is not made. Instead, the peritoneum is entered through direct cutdown, video-assisted trocar insertion, or Veress needle insertion for initial pneumoperitoneum followed by trocar insertion. Peritoneum access was chosen based on surgeon preference. After the initial port placement, the remaining three to four ports were placed under direct visualization aimed at the target anatomy. Once all ports were in, the robot was docked similar to above. The gallbladder was removed through a $10-\mathrm{mm}$ port. The fascia defect of the 10 -mm port sites was closed.

\section{Results}

Among the 104 SIRC cases, 78 (75\%) were women and 26 (25\%) were men, and among the 105 MIRC cases, 75 (71.4\%) were women and 30 (28.6\%) were men. The average age in SIRC was 53 years and MIRC was 52 years. There was no significance in the age selection for each approach.

We found that SIRC had a less operative time, $(M=94.0$ minutes, $S E=20.9)$ compared to $M I R C(M=99.9$ minutes, $\mathrm{SE}=30.3)$, and the EBL was less with single-incision $(\mathrm{M}=11.52 \mathrm{~mL}, \mathrm{SE}=9.717)$ vs multi-incision $(\mathrm{M}=17.17 \mathrm{~mL}, \mathrm{SE}=23.686)$ by a significant margin $(p=0.016 \& p=0.004$ respectively). Console time though was not significantly less $(p=0.370)$ for SIRC $(\mathrm{M}=41.1$ minutes, $\mathrm{SE}=16.0)$ vs. MIRC $(\mathrm{M}=44.7$ minutes, $\mathrm{SE}=$ 9.1; Tables 1 \& 2). 


\section{Cureus}

\begin{tabular}{|c|c|c|c|c|c|}
\hline & Arm & $\mathbf{N}$ & Mean & Standard Deviation & Standard Error Mean \\
\hline \multirow[t]{2}{*}{ Console time } & SIRC & 98 & 41.10 & 15.941 & 1.610 \\
\hline & MIRC & 93 & 44.70 & 19.131 & 1.984 \\
\hline \multirow[t]{2}{*}{ Operation time } & SIRC & 104 & 94.04 & 20.931 & 2.052 \\
\hline & MIRC & 105 & 99.89 & 30.225 & 2.950 \\
\hline \multirow[t]{2}{*}{ Age } & SIRC & 104 & 53.16 & 18.350 & 1.799 \\
\hline & MIRC & 105 & 51.99 & 18.330 & 1.789 \\
\hline \multirow{2}{*}{ Estimated blood loss } & SIRC & 104 & 11.52 & 9.717 & 0.957 \\
\hline & MIIRC & 105 & 17.17 & 23.686 & 2.311 \\
\hline
\end{tabular}

TABLE 1: Single-incision robotic cholecystectomy and multi-incision robotic cholecystectomy findings

SIRC, single-incision robotic cholecystectomy; MIRC, multi-incision robotic cholecystectomy

\begin{tabular}{|c|c|c|c|}
\hline & SIRC & MIRC & P-value \\
\hline Console time (min) & $41.1+/-16.0$ & $44.7+/-19.1$ & 0.370 \\
\hline Operative time (min) & $94.0+/-20.9$ & $99.9+/-30.3$ & $0.016^{\star}$ \\
\hline Age (years) & $53.16+/-18.35$ & $51.99+/-18.33$ & 0.644 \\
\hline Estimated blood loss (mL) & $11.52+/-9.717$ & $17.17+/-23.686$ & $0.026^{\star}$ \\
\hline
\end{tabular}

TABLE 2: Statistical analysis of single-incision robotic cholecystectomy and multi-incision robotic cholecystectomy findings

*indicates significance; SIRC, single-incision robotic cholecystectomy; MIRC, multi-incision robotic cholecystectomy

Both symptomatic cholelithiasis and dyskinesia were identified to be the most common indicators for surgical intervention in 36 cases (34.6\%) of SIRC. Among MIRC cases, symptomatic cholelithiasis was the most common indicator 53 (50.5\%) cases, followed by dyskinesia in 25 (23.8\%) cases. This suggests that dyskinesia was observed in approximately one-third of SIRC and only one-fourth of MIRC cases. Acute and chronic cholecystitis were relatively minimal indicators for the surgery: acute observed in three (3.0\%) cases and chronic in seven (6.7\%) cases of SIRC, while acute in six (5.7\%) and chronic in four (3.8\%) cases of MIRC (Table 3). 


\section{Cureus}

\begin{tabular}{lll}
\hline Indication & SIRC & MIRC \\
\hline Dyskinesia & $36(34.6 \%)$ & $25(23.8 \%)$ \\
Symptomatic cholelithiasis & $36(34.6 \%)$ & $53(50.5 \%)$ \\
Acute cholecystitis & $3(2.9 \%)$ & $6(5.7 \%)$ \\
Chronic cholecystitis & $7(6.7 \%)$ & $4(3.8 \%)$ \\
Unknown & $16(15.4 \%)$ & $11(10.5 \%)$ \\
Others (choledocholithiasis, polyp, etc.) & $6(5.8 \%)$ & $6(5.7 \%)$
\end{tabular}

TABLE 3: Single-incision robotic cholecystectomy and multi-incision robotic cholecystectomy indication for operation

SIRC, single-incision robotic cholecystectomy; MIRC, multi-incision robotic cholecystectomy

Intraoperatively, 45 (42.9\%) cases of MIRC received irrigation usually for bile spillage, while 31 (29.8\%) cases of SIRC received irrigation for the same reason. The use of firefly visualization use was similar in both cases: 22 (21\%) cases of MIRC and 21 (20.2\%) of SIRC. Although uncommon, a gallbladder perforation with leakage of bile was noticed in $15(14.3 \%)$ cases of MIRC and 18 (17.3\%) of SIRC. Intraoperative cholangiogram was performed in only seven (6.67\%) cases of MIRC and eight (7.70\%) of SIRC (Table 4). The LOS parameter comprised outpatients in 75 (71.4\%) cases of MIRC and 84 (80.8\%) SIRC (Table 5). Using Pearson chi-square analysis, there was a significant difference in the use of irrigation and LOS between SIRC and MIRC (Table 6). All other intraoperative events did not significantly differ between the two approaches as the null is rejected.

\begin{tabular}{|c|c|c|}
\hline Intraop Events & SIRC & MIRC \\
\hline Irrigation & $31(29.8 \%)$ & 45 (42.9\%) \\
\hline Firefly usage & $21(20.2 \%)$ & $22(21.0 \%)$ \\
\hline Perforation & $18(17.3 \%)$ & $15(14.3 \%)$ \\
\hline Intraoperative cholangiogram & $8(7.70 \%)$ & $7(6.67 \%)$ \\
\hline
\end{tabular}

TABLE 4: Single-incision robotic cholecystectomy and multi-incision robotic cholecystectomy intraop events

SIRC, single-incision robotic cholecystectomy; MIRC, multi-incision robotic cholecystectomy

\begin{tabular}{|c|c|c|}
\hline Length of stay & SIRC & MIRC \\
\hline 0 days & 89 (80.8\%) & 75 (71.4\%) \\
\hline 1 day & 7 (14.4\%) & 17 (16.2\%) \\
\hline >1 day & $8(7.70 \%)$ & 13 (12.4\%) \\
\hline \multicolumn{3}{|c|}{$\begin{array}{l}\text { TABLE 5: Single-incision robotic cholecystectomy and multi-incision robotic cholecystectom } \\
\text { length of stay }\end{array}$} \\
\hline \multicolumn{3}{|c|}{ SIRC, single-incision robotic cholecystectomy; MIRC, multi-incision robotic cholecystectomy } \\
\hline
\end{tabular}




\section{Cureus}

\begin{tabular}{|l|l|}
\hline Pearson Chi-square & P-value \\
\hline Indication & 0.1579 \\
\hline Irrigation & $0.0499^{*}$ \\
Firefly & 0.895 \\
\hline Perforation & 0.549 \\
\hline Intraoperative cholangiogram & 0.773 \\
\hline Length of stay & $0.0379^{*}$ \\
\hline
\end{tabular}

\section{TABLE 6: Single-incision robotic cholecystectomy and multi-incision robotic cholecystectomy statistical analysis of intraop events}

*indicates a significant difference between the two approaches

No cases reviewed required conversion to open or laparoscopic approaches as these were excluded. None of the patients suffered bile duct or any other injuries to surrounding structures. Intraoperatively, there was one case of bowel enterotomy with trocar placement in the multi-incision approach. There was one report of gallbladder enterotomy with gross spillage of stones with bile. Umbilical hernias were found and repaired in three $(2.88 \%)$ cases of SIRC.

\section{Discussion}

Robotic surgery is becoming more common, tripling over laparoscopic surgery across all disciplines in surgery from $6.8 \%$ in 2008 to $17 \%$ in 2012 [6]. SIRCs and MIRCs have shown promising results as the most important cholecystectomies. This is supported by the low complication rate due to the control afforded to the surgeon. When comparing MIRC to SIRC in the first nine children to receive elective robotic cholecystectomy in a tertiary-care institution (by a single surgeon), SIRC had a greater total operative time (SIRC 169 minutes vs MIRC 139 minutes), with the difference between the medians being 30 minutes, and greater total console time (69 minutes SIRC vs 47 minutes MIRC), with the difference being 22 minutes [4]. This is quite contradictory to our data, which may be attributed to the learning curve associated with SIRCs compared to MIRCs as well as the experience from the nine surgeons in this study compared to the single surgeon in the tertiary-care institution. Comparing single- and multi-incision approaches for hysterectomies for endometrial cancer, there was a significance difference in blood loss between the two approaches (50 mL single-incision vs $100 \mathrm{~mL}$ multi-incision) and total hospital stay (two days singleincision hysterectomy vs three days multi-incision hysterectomy), while the total operative time has been found to be similar among the two (110 minutes single-incision hysterectomy vs 102.5 minutes multiincision hysterectomy) [7]. Our results affirm that single-incision has less total hospital stay, likely attributed to the avoidance of vasculature with the single incision residing in midline while multi-incision incisions made away from the midline, the risk being placing through an inferior epigastric or another blood vessel.

Laparoscopic approaches, on the other hand, do not offer much difference in outcomes except the roboticassisted cholecystectomy has been associated with less biliary injuries due to the higher precision [8]. We encountered no identified biliary injuries in our review of 209 patients over five years. The higher precision of robotic surgery has been known since its introduction, but the full feasibility comes in the narrow anatomical areas of the body where the surgeon has the option to adjust the console to robotic instrument movement ratios. Laparoscopic approach to cholecystectomy was found to be inferior to a robotic-assisted approach requiring more conversion, longer operative time, more perioperative bile spillage, and more postop bile spillage. Although the power of these findings remained of low non-significant value, early suggestion of these variables is important to note and follow in the future [3]. Pain level has been found to be less with the robotic approach when compared to laparoscopic approach [7]. This decrease in pain may be related to the focus of the robotic system to place the center of rotation and movement at the patients' fascia, reducing the overall stress forces encountered in the operation. In comparing robotic-assisted abdomino-perineal resections (APR) vs laparoscopic approach, the robotic approach allowed for better visualization, given the enhanced three-dimensional visualization and precision of the EndoWrist [1,3,9-10].

Driven by a lack of concrete evidence to the benefits of SIRC and MIRC, we conducted a retrospective chart review comparing the clinical and perioperative outcomes of SIRC and MIRC. Our comparison consisted of 209 patients, 105 MIRC and 104 SIRC cases, retrospectively, in a community hospital. In addition to reviewing the outcomes, we compared operative time, console time, EBL, and any significant intraoperative events and matched these based on patient age and gender. Choice of an SIRC vs MIRC approach was 
determined by surgeon preference, but SIRC compared to laparoscopic approach is generally favored for patients of lower BMIs [2].

Our report indicates that SIRC has advantages over MIRC including less EBL, supported by previous articles, and less total operative time. We did not find any significant difference in total console time between the two approaches. Intraoperatively, symptomatic cholelithiasis was the overall primary indicator for both SIRC and MIRC cases, with dyskinesia being the second most common indicator, although observed in only $36.4 \%$ cases of SIRC and $23.8 \%$ of MIRC. Symptomatic cholelithiasis was the primary indicator for $50.5 \%$ of MIRC and only $36.4 \%$ of SIRC. It is assumed that SIRC patients are not in acute distress and have time for the cosmetically superior SIRC approach, as supported by $36.4 \%$ of the SIRC patients having stable dyskinesia vs only $23.8 \%$ or MIRC [2]. Intraoperative events did not vary among the two approaches and only irrigation usage was significant only marginally, which is most likely due to surgeon preference. Three SIRC cases had umbilical hernias identified during operation, and no follow-up regarding postop hernias was recorded, but previous articles found a $7.1 \%$ occurrence of ventral hernias following SIRC with an average of 27.7 months postop [2]

Our data indicate that the hospital LOS remained lower for SIRC cases, thus reducing the expenditure of SIRC to some extent; however, the instruments and set up involved in SIRC contribute to the high cost when compared to MIRC: perioperative cost SIRC $\$ 6158$ vs MIRC $\$ 4288$ USD $(p<0.0001)$ [2]. Pediatric patients undergoing SIRC, when compared against a laparoscopic approach, have half the hospital stay [5]. This cost does not include capital acquisition, team training, maintenance of equipment and repair, and operating room setup time. By saving on the hospital stay, the overall savings outweigh the intraoperative cost. In comparing robotic versus open common bile duct exploration, a net saving of $\$ 3000$ per case was noted [9]. This is also associated with less hospital-acquired complications with shorter inhospital stay.

\section{Conclusions}

SIRC and MIRC cases are both safe and feasible ways to remove the inflamed/dysfunctional gallbladder. SIRC is associated with less operative time, less blood loss, and shorter hospital stay. Further large-scale studies are needed to make a definitive decision as many of the variables were not significant possibly due to the small single-institution sample size. Although the robotic approach should be individualized based on the surgeon experience/preference, according to our study, SIRCs seemed to have improved outcomes.

\section{Additional Information \\ Disclosures}

Human subjects: Consent was obtained by all participants in this study. Easton Hospital IRB issued approval Not applicable. Easton Hospital IRB approval of Comparison of intraoperative outcomes between single-incision robotic cholecystectomy and multi-incision robotic cholecystectomy . Animal subjects: All authors have confirmed that this study did not involve animal subjects or tissue. Conflicts of interest: In compliance with the ICMJE uniform disclosure form, all authors declare the following: Payment/services info: All authors have declared that no financial support was received from any organization for the submitted work. Financial relationships: All authors have declared that they have no financial relationships at present or within the previous three years with any organizations that might have an interest in the submitted work. Other relationships: All authors have declared that there are no other relationships or activities that could appear to have influenced the submitted work.

\section{References}

1. Yarbrough MF, Misra S: Robotic-assisted abdomino-perineal resection: efficacy and safety in a community hospital setting. World J Color Surg. 2016, 6:1-17.

2. Hagen ME, Balaphas A, Podetta M, et al.: Robotic single-site versus multiport laparoscopic cholecystectomy: a case-matched analysis of short- and long-term costs. Surg Endosc. 2018, 32:1550-1555. 10.1007/s00464-017-5843-z

3. Su WL, Huang JW, Wang SN, et al.: Comparison study of clinical outcomes between single-site robotic cholecystectomy and single incision laparoscopic cholecystectomy. Asian J Surg. 2017, 40:424-428. 10.1097/MD.0000000000012103

4. N. Ahn, G. Signor, T. P. Singh, S. Stain, and C. Whyte: Robotic single- and multisite cholecystectomy in children. J Laparoendosc Adv Surg Tech. 2015, 25:1033-1035. 10.1089/lap.2017.0532

5. P. Mattei: Single-site robotic-assisted laparoscopic cholecystectomy in children and adolescents: a report of 20 cases. Surg Endosc. 2018, 32:2402-2408. 10.1007/s00464-017-5939-5

6. Juo YY, Mantha A, Abiri A, Lin A, Dutson E: Diffusion of robotic-assisted laparoscopic technology across specialties: a national study from 2008 to 2013. Surg Endosc. 2018, 32:1405-1413. 10.1111/bju.12247

7. Corrado G, Cutilo E, Mancini E, et. al.: Robotic single site versus robotic multiport hysterectomy in early endometrial cancer: a case control study. J Gynecol Oncol. 2016, 27:e39. 10.3802/jgo.2016.27.e39

8. Ayloo S, Roh Y, Choudhary N: Laparoscopic versus robot-assisted cholecystectomy: a retrospective cohort study. Int J Surg. 2014, 12:1077-81. 10.1016/j.ijsu.2014.08.405

9. Almamar A, Alkhamesi NA, Davies WT, Schlachta CM: Cost analysis of robot-assisted choledochotomy and common bile duct exploration as an option for complex choledocholithiasis. Surg Endosc. 2018, 32:12231227. 10.1007/s00464-017-5795-3 


\section{Cureus}

10. Kim S, May A, Ryan H, Mohsin A, Tsuda S: Distraction and proficiency in laparoscopy: 2D versus robotic console 3D immersion. Surg Endosc. 2017, 31:4625-4630. 10.1007/s00464-017-5525-X 